\title{
ON SOME TRIGONOMETRIC SUMMABILITY METHODS AND GIBBS' PHENOMENON
}

\author{
BY \\ OTTO SZÁSZ
}

1. Introduction. We consider Fourier sine series

$$
f(\theta) \sim \sum_{1}^{\infty} b_{\nu} \sin \nu \theta, \quad \sum_{1}^{n} b_{\nu} \sin \nu \theta=s_{n}(\theta) .
$$

Suppose that $f(+0)$ exists and is greater than 0 , that is $f(\theta)$ has a simple jump $2 f(+0)$ at $\theta=0$. We say that the series (1.1) presents Gibbs' phenomenon, if

$$
\lim \sup s_{n}\left(\theta_{n}\right)>f(+0), \text { as } \theta_{n} \downarrow 0 .
$$

More generally, if only for some $k \geqq 0$

$$
\lim _{\theta+0} \frac{2(k+1)}{\theta^{k+1}} \int_{0}^{\theta}(\theta-t)^{k} f(t) d t=j>0
$$

exists, and $\lim \sup s_{n}\left(\theta_{n}\right)>j / 2$, we say that the series (1.1) presents a generalized Gibbs' phenomenon at $\theta=0 ; j$ is the generalized jump of $f(\theta)$ at $\theta=0$. Our aim is to find general conditions for $f(\theta)$ lor its Fourier coefficients, which imply a Gibbs' phenomenon. It is known that the jump $j$ is closely connected with the asymptotic behavior of the sequence $\left\{n b_{n}\right\}$; on the other hand

$$
s_{n}\left(\theta_{n}\right)=\sum_{1}^{n} \nu b_{\nu} \frac{\sin \nu \theta_{n}}{\nu}
$$

is a linear transform of $\left\{n b_{n}\right\}$, with the triangular matrix $a_{n \nu}=\nu^{-1} \sin \nu \theta_{n}$, $\nu=1,2, \cdots, n$. In a previous paper $[3]\left({ }^{1}\right)$ we have discussed the relationship of the transform

$$
T_{n}\left(\theta_{n}\right)=\sum_{1}^{n} \tau_{\nu} \frac{\sin \nu \theta_{n}}{\nu}
$$

to the Cesàro means of the sequence $\left\{\tau_{n}\right\}$. As an application we have proved the presence of a Gibbs' phenomenon under general assumptions and we gave new formulae for the determination of the jump $2 f(+0)$. More general results are given in the present paper; the knowledge of (3) is not assumed.

We consider trigonometric linear transforms, related to $T_{n}$. We discuss in particular the transforms

Presented to the Society, April 24, 1943; received by the editors April 24, 1943.

(1) Numbers in brackets refer to the literature cited at the end of this paper. 


$$
\begin{gathered}
B_{n}=\frac{1}{2}\left\{T_{n}\left(\theta_{n}\right)+T_{n}\left(\phi_{n}\right)\right\}, \quad H_{n}=\frac{1}{n} \sum_{k=1}^{n} T_{\kappa}\left(\theta_{\kappa}\right), \\
S_{n}=\frac{1}{\theta_{n}} \int_{0}^{\theta_{n}} T_{n}(t) d t .
\end{gathered}
$$

In $\$ 5$ we prove a theorem on $(C, 2)$ summability of the sequence $\left\{n b_{n}\right\}$ for Fourier series.

We shall make repeated use of the well known theorem:

THEOREM A. The convergence of a sequence $\left\{\tau_{n}\right\}$ implies the convergence of the transform $T_{n}=\sum_{1}^{n} a_{n v} \tau_{v}$, if and only if

$$
\begin{aligned}
\lim _{n \rightarrow \infty} a_{n \nu} & =a_{\nu} \text { exists for } \nu=1,2,3, \cdots, \\
\sum_{\nu=1}^{n}\left|a_{n \nu}\right| & =O(1), \text { as } n \rightarrow \infty, \\
\lim _{n \rightarrow \infty} \sum_{\nu=1}^{n} a_{n \nu} & =\sigma \text { exists. }
\end{aligned}
$$

We then have $\lim T_{n}=\sigma \lim \tau_{n}+\sum_{1}^{\infty} a_{\nu}\left(\tau_{\nu}-\lim \tau_{n}\right)\left({ }^{2}\right)$.

2. The average $(1 / 2)\left\{T_{n}\left(\theta_{n}\right)+T_{n}\left(\phi_{n}\right)\right\}=B_{n}$. On putting $\rho_{n}=1$ in Theorem 2 of our paper [3] we get easily the following theorem.

\section{THEOREM 1. Suppose that}

$$
\frac{1}{n} \sum_{1}^{n} \tau_{\nu} \rightarrow \tau, \quad \phi_{n}>0, \quad \theta_{n} \geqq 0, \quad n \theta_{n}=O(1), \quad n \phi_{n}=O(1),
$$

and that $\sigma(\alpha)=\int_{0}^{\alpha}\left(t^{-1} \sin t\right) d t$ has the same value for all limit points $\alpha$ of the sequence $\left\{n \theta_{n}\right\}$, and the same value for all limit points $\beta$ of the sequence $\left\{n \phi_{n}\right\}$; then

$$
\lim _{n \rightarrow \infty} B_{n}=(\tau / 2)(\sigma(\alpha)+\sigma(\beta)) .
$$

For the special case $\phi_{n}=\pi / n$ see Rogosinski $[1,2]$.

We next assume $(C, 2) \lim \tau_{n}=\tau$. Let $\tau_{n}^{\prime}=\sum_{1}^{n} \tau_{\nu}, \tau_{n}^{2}=\sum_{1}^{n} \tau_{\nu}^{1}$, then our assumption is: $2 \tau_{n}^{2} / n^{2} \rightarrow \tau$. We write $\Delta^{0} \tau_{n}=\tau_{n}, \Delta \tau_{n}=\tau_{n}-\tau_{n+1}, \Delta^{2} \tau_{n}=\Delta\left(\Delta \tau_{n}\right)$; then

$$
\begin{aligned}
B_{n}= & \sum_{1}^{n-2} \tau_{\nu}^{2} \Delta^{2} \frac{\sin \nu \theta_{n}+\sin \nu \phi_{n}}{\nu}+\tau_{n-1}^{2}\left\{\frac{\sin (n-1) \theta_{n}+\sin (n-1) \phi_{n}}{n-1}\right. \\
& \left.-2 \frac{\sin n \theta_{n}+\sin n \phi_{n}}{n}\right\}+\tau_{n}^{2} \frac{\sin n \theta_{n}+\sin n \phi_{n}}{n} .
\end{aligned}
$$

(2) The statement at the beginning of $\$ 2$ in [3] should be corrected accordingly; it does not affect the rest of the paper. 
It follows from Theorem A that, if in addition to the assumptions of Theorem 1,

$$
\begin{gathered}
\sum_{1}^{n-2} \nu^{2}\left|\Delta^{2} \frac{\sin \nu \theta_{n}+\sin \nu \phi_{n}}{\nu}\right|+n^{2}\left|\Delta \frac{\sin (n-1) \theta_{n}+\sin (n-1) \phi_{n}}{n-1}\right| \\
+n\left|\sin n \theta_{n}+\sin n \phi_{n}\right|=O(1),
\end{gathered}
$$

then again (2.2) holds. Then

We now assume $n\left(\phi_{n}-\theta_{n}\right)=(2 \mu-1) \pi+\delta_{n}, \delta_{n}=O(1 / n), \mu$ an integer $\geqq 1$.

$$
\sin n \theta_{n}+\sin n \phi_{n}=2 \sin \frac{n}{2}\left(\theta_{n}+\phi_{n}\right) \cos \frac{n}{2}\left(\theta_{n}-\phi_{n}\right)=o\left(\frac{1}{n}\right)
$$

also

$$
\begin{aligned}
\cos \frac{n-1}{2}\left(\theta_{n}-\phi_{n}\right)= & \cos \frac{n}{2}\left(\theta_{n}-\phi_{n}\right) \cos \frac{\theta_{n}-\phi_{n}}{2} \\
& +\sin \frac{n}{2}\left(\theta_{n}-\phi_{n}\right) \sin \frac{\theta_{n}-\phi_{n}}{2}=O\left(\frac{1}{n}\right) .
\end{aligned}
$$

Furthermore, for $\theta>0$,

Hence

$$
\Delta^{2}\left(\frac{\sin \nu \theta}{\nu}\right)=\Delta^{2} \int_{0}^{\theta} \cos \nu t d t=R \int_{0}^{\theta} \Delta^{2} z^{\nu} d t, \quad z=c^{i t} .
$$

and

$$
\Delta^{2} \frac{\sin \nu \theta}{\nu}=R \int_{0}^{\theta} z^{\nu}(1-z)^{2} d t
$$

$$
\begin{aligned}
\left|\Delta^{2} \frac{\sin \nu \theta}{\nu}\right| & <\int_{0}^{\theta}|1-z|^{2} d t=\int_{0}^{\theta}\left|e^{-i t / 2}-c^{i t / 2}\right|^{2} d t \\
& =4 \int_{0}^{\theta} \sin ^{2} \frac{t}{2} d t<\int_{0}^{\theta} t^{2} d t=\frac{\theta^{3}}{3} .
\end{aligned}
$$

Thus in view of (2.1)

$$
\sum_{1}^{n-2} \nu^{2}\left|\Delta^{2} \frac{\sin \nu \theta_{n}+\sin \nu \phi_{n}}{\nu}\right|<\left(\theta_{n}^{8}+\phi_{n}^{3}\right) \sum_{1}^{n} \nu^{2}=O(1) .
$$

We have thus proved the following theorem.

Theorem 2. Suppose that $(C, 2) \lim \tau_{n}=\tau, \phi_{n}>0, \theta_{n} \geqq 0, n \theta_{n}=O(1)$, $n \phi_{n}=n \theta_{n}+(2 \mu-1) \pi+O(1 / n), \mu$ an integer greater than or equal to 1 , and that $\sigma(\alpha)=\int_{0}^{\alpha} t^{-1} \sin t d t$ has the same value for all limit points $\alpha$ of the sequence $\left\{n \theta_{n}\right\}$. Then $\lim B_{n}=(\tau / 2)\{\sigma(\alpha)+\sigma(\alpha+(2 \mu-1) \pi)\}$.

3. The average $(1 / n) \sum_{k=1}^{n} T_{\kappa}\left(\theta_{\kappa}\right)=H_{n}$. We have 


$$
n H_{n}=\sum_{k=1}^{n} \sum_{\nu=1}^{\kappa} \nu^{-1} \tau_{\nu} \sin \nu \theta_{k}=\sum_{\nu=1}^{n} \nu^{-1} \tau_{\nu} \sum_{k=\nu}^{n} \sin \nu \theta_{\kappa}=\sum_{\nu=1}^{n} C_{n \nu} \tau_{\nu}
$$

where

$$
C_{n \nu}=\nu^{-1} \sum_{\kappa=\nu}^{n} \sin \nu \theta_{\kappa}, \quad \nu=1,2, \cdots, n .
$$

Thus $n H_{n}=\sum_{1}^{n-1}\left(C_{n \nu}-C_{n, \nu+1}\right) \tau_{\nu^{\prime}}+C_{n n} \tau_{u}^{\prime}$. We first prove the following theorem.

Theorem 3. If $(C, 1) \lim \tau_{n}=\tau$, and

$$
\frac{1}{n} \sum_{1}^{n}\left|\nu \theta_{\nu}-\alpha\right| \rightarrow 0 \text {, }
$$

then

$$
H_{n} \rightarrow \tau \int_{j}^{\alpha} \frac{\sin t}{t} d t
$$

(3.3) is strong summability $(C, 1)$ of the sequence $\left\{n \theta_{n}\right\}$ to $\alpha$; it implies that $\theta_{n} \rightarrow 0$, hence $\lim _{n \rightarrow \infty} n^{-1} C_{n \nu}=0$ for each $\nu$. Furthermore

$$
\begin{aligned}
\sum_{1}^{n-1} \nu\left|C_{n \nu}-C_{n, \nu+1}\right| & +n\left|C_{n n}\right| \\
= & \sum_{1}^{n-1}\left|\sum_{k=\nu}^{n} \sin \nu \theta_{k}-\frac{\nu}{\nu+1} \sum_{\nu+1}^{n} \sin (\nu+1) \theta_{k}\right|+\left|\sin n \theta_{n}\right| \\
& <\sum_{1}^{n-1}\left|\sin \nu \theta_{\nu}\right|+\sum_{1}^{n-1}\left|\sum_{k=\nu+1}^{n}\left(\sin \nu \theta_{k}-\sin (\nu+1) \theta_{k}\right)\right| \\
& +\sum_{1}^{n-1} \frac{1}{\nu+1}\left|\sum_{\nu+1}^{n} \sin (\nu+1) \theta_{k}\right|+1 \\
& <\sum_{1}^{n} \nu \theta_{\nu}+2 \sum_{1}^{n-1} \sum_{\nu+1}^{n}\left|\sin \frac{\theta_{k}}{2} \cos \frac{2 \nu+1}{2} \theta_{k}\right|+\sum_{1}^{n-1} \sum_{\nu+1}^{n} \theta_{k}+1 \\
& <3 \sum_{1}^{n} \nu \theta_{\nu}+1=O(n), \quad \text { as } n \rightarrow \infty,
\end{aligned}
$$

hence $H_{n}{ }^{\circ}=O(1)$. Thus, in order that $H_{n}$ has a limit whenever $n^{-1} \tau_{n}^{\prime}$ tends to a $\operatorname{limit} \tau$, the additional condition is that

$$
\lim _{n \rightarrow \infty} \frac{1}{n} \sum_{\nu=1}^{n} C_{n \nu}=\lim \frac{1}{n} \sum_{\nu=1}^{n} \nu^{-1} \sum_{\alpha=\nu}^{n} \sin \nu \theta_{\alpha}=\sigma
$$

exists. We then have $H_{n} \rightarrow \sigma \tau$ (cf. Theorem A). Now 
and

$$
\frac{1}{n} \sum_{1}^{n} C_{n \nu}=\frac{1}{n} \sum_{k=1}^{n} S_{k}\left(\theta_{k}\right)
$$

Furthermore

$$
\begin{aligned}
S_{k}\left(\theta_{k}\right) & =\sum_{\lambda=1}^{k} \lambda^{-1} \sin \lambda \theta_{k}=\int_{0}^{\theta_{k}}\left(\sum_{1}^{k} \cos \lambda t\right) d t \\
& =-\frac{\theta_{k}}{2}+\int_{0}^{\theta_{k}} \frac{\sin (\kappa+1 / 2) t}{2 \sin (t / 2)} d t .
\end{aligned}
$$

$$
\begin{aligned}
\int_{0}^{\theta} \frac{\sin (\kappa+1 / 2) t}{2 \sin (t / 2)} d t=\int_{0}^{\theta} \frac{\sin (\kappa+1 / 2) t}{t} d t & \\
& \quad+\int_{0}^{\theta} \sin (\kappa+1 / 2) t\left\{\frac{1}{2 \sin (t / 2)}-\frac{1}{t}\right\} d t
\end{aligned}
$$

and, from the mean value theorem,

$$
0<\frac{1}{2 \sin (t / 2)}-\frac{1}{t}=\frac{t-2 \sin (t / 2)}{2 t \sin (t / 2)}<\frac{t}{12}, \quad \theta<t<\pi .
$$

Hence

$$
\left|\int_{0}^{\theta} \sin (\kappa+1 / 2) t\left\{\frac{1}{2 \sin (t / 2)}-\frac{1}{t}\right\} d t\right|<\frac{1}{12} \int_{0}^{\theta} t d t=\frac{1}{24} \theta^{2},
$$

and

$$
\begin{aligned}
S_{\kappa}\left(\theta_{\kappa}\right) & =-\frac{\theta_{\kappa}}{2}+\int_{0}^{(\kappa+1 / 2) \theta_{k}} \frac{\sin t}{t} d t+\delta_{\kappa} \theta_{\kappa}^{2} \\
& =\int_{0}^{\alpha} \frac{\sin t}{t} d t+\int_{\alpha}^{(\kappa+1 / 2) \theta_{k}} \frac{\sin t}{t} d t-\frac{\theta_{k}}{2}+\delta_{\kappa} \theta_{k}^{2},
\end{aligned}
$$

where $\left|\delta_{k}\right|<1 / 24$. Thus, for $0<\theta_{k}<1$,

$$
\left|S_{\kappa}\left(\theta_{\kappa}\right)-\int_{0}^{\alpha} \frac{\sin t}{t} d t\right|<\left|(\kappa+1 / 2) \theta_{\kappa}-\alpha\right|+\theta_{\kappa}<\left|\kappa \theta_{\kappa}-\alpha\right|+2 \theta_{\kappa},
$$

and from (3.3)

$$
\left|\frac{1}{n} \sum_{1}^{n} S_{\kappa}\left(\theta_{\kappa}\right)-\int_{0}^{\alpha} \frac{\sin t}{t} d t\right|<\frac{1}{n} \sum_{1}^{n}\left|\kappa \theta_{\kappa}-\alpha\right|+\frac{2}{n} \sum_{1}^{n} \theta_{\kappa} \rightarrow 0 ;
$$

which proves Theorem 3. For the case of $(C, 2)$ summability we prove the following theorem.

THEOREM 4. If $(C, 2) \lim \tau_{n}=\tau$, 


$$
\sum_{1}^{n} \nu^{2}\left|\theta_{v}-\theta_{v+1}\right|=O(n), \quad \text { as } n \rightarrow \infty,
$$

and if (3.3) holds, then (3.4) holds.

Evidently (3.3) implies $\sum_{1}^{n} \nu \theta_{\nu}=O(n)$; furthermore

$$
\sum_{1}^{n} \nu^{2}\left(\theta_{\nu}-\theta_{\nu+1}\right)=\sum_{1}^{n}(2 \nu-1) \theta_{\nu}-n^{2} \theta_{n+1}=O(n)-n^{2} \theta_{n+1},
$$

hence from (3.6)

$$
n \theta_{n}=O(1) \text { as } n \rightarrow \infty
$$

We have from (3.1)

$$
n H_{n}=\sum_{1}^{n-2} \tau_{\nu}^{\prime \prime} \Delta^{2} C_{n \nu}+\tau_{n-1}^{\prime \prime}\left(C_{n, n-1}-2 C_{n n}\right)+\tau_{n}^{\prime \prime} C_{n n},
$$

where $n C_{n n}=\sin n \theta_{n}=O(1)$, and

$n\left(C_{n, n-1}-2 C_{n n}\right)=O(1)+(n /(n-1))\left\{\sin (n-1) \theta_{n-1}+\sin (n-1) \theta_{n}\right\}=O(1)$.

Furthermore, using (2.4) and (3.8),

$$
\begin{aligned}
& \sum_{1}^{n-2} \nu^{2}\left|\Delta^{2} C_{n \nu}\right| \\
& =\sum_{1}^{n-2} \nu^{2}\left|\frac{1}{\nu} \sum_{k=\nu}^{n} \sin \nu \theta_{k}-\frac{2}{\nu+1} \sum_{k=\nu+1}^{n} \sin (\nu+1) \theta_{k}+\frac{1}{\nu+2} \sum_{k=\nu+2}^{n} \sin (\nu+2) \theta_{k}\right| \\
& \leqq \sum_{1}^{n-2} \nu^{2}\left|\frac{1}{\nu}\left(\sin \nu \theta_{\nu}+\sin \nu \theta_{\nu+1}\right)-\frac{2}{\nu+1} \sin (\nu+1) \theta_{\nu+1}\right| \\
& \quad+\sum_{1}^{n-2} \nu^{2}\left|\sum_{k=\nu+2}^{n}\left(\frac{\sin \nu \theta_{k}}{\nu}-\frac{2 \sin (\nu+1) \theta_{k}}{\nu+1}+\frac{\sin (\nu+2) \theta_{k}}{\nu+2}\right)\right| \\
& <O(n)+\sum_{1}^{n-1} \nu\left|\sin \nu \theta_{\nu}+\sin \nu \theta_{\nu+1}-2 \sin (\nu+1) \theta_{\nu+1}\right| \\
& \quad+\sum_{1}^{n-2} \nu^{2} \sum_{k=\nu+2}^{n}\left|\Delta^{2} \nu^{-1} \sin \nu \theta_{k}\right| \\
& <O(n)+\sum_{1}^{n-1} \nu\left(\theta_{\nu+1}+\theta_{\nu+1}+\nu\left|\theta_{\nu}-\theta_{\nu+1}\right|\right)+\sum_{1}^{n} \nu^{2}\left(\sum_{k=\nu}^{n} \theta_{k}^{3}\right) \\
& =O(n)+O\left(\sum_{1}^{n} \nu^{3} \theta_{\nu}^{3}\right)=O(n) .
\end{aligned}
$$


Now (3.4) follows as in Theorem 3. This proves Theorem 4. In this connection the following lemma is of interest:

LEMMA. If for some $c>0$

$$
0 \leqq \theta_{n+1} \leqq(1+c / n) \theta_{n}, \quad n=1,2,3, \cdots,
$$

and

$$
\sum_{1}^{n} \nu \theta_{\nu}=O(n)
$$

as $n \rightarrow \infty$,

then (3.6) and (3.8) hold.

We write $\sum_{1}^{n} \nu^{2}\left|\theta_{\nu}-\theta_{\nu+1}\right|=\sum^{\prime}+\sum^{\prime \prime}$, where $\sum^{\prime}$ contains all terms with $\theta_{\nu} \leqq \theta_{\nu+1}$, and $\sum^{\prime \prime}$ the rest. Now, using (3.9) and (3.10),

$$
\sum^{\prime} \leqq c \sum_{1}^{n} \nu \theta_{\nu}=O(n)
$$

and from (3.7)

$$
\sum^{\prime \prime}-\sum^{\prime}<\sum_{1}^{n}(2 \nu-1) \theta_{\nu}=O(n),
$$

hence $\sum^{\prime \prime}=O(n)$ and (3.6) is proved. This and (3.10) yield (3.8). In particular in Theorem 4 assumption (3.6) can be replaced by $\theta_{n} \downarrow$.

4. The integral mean $\left(1 / \theta_{n}\right) \int_{0}^{\theta_{n}}\left(\sum_{1}^{n} \tau_{\nu} \nu^{-1} \sin \nu t\right) d t$. To a given sequence $\left\{\tau_{n}\right\}$ we consider the transform

$$
S_{n}=\frac{1}{\theta_{n}} \int_{0}^{\theta_{n}}\left(\sum_{1}^{n} \tau_{\nu} \sin \nu t\right) d t=\sum_{1}^{n} \frac{1-\cos \nu \theta_{n}}{\nu^{2} \theta_{n}} \tau_{\nu}=\sum_{1}^{n} a_{n \nu} \tau_{\nu}, \theta_{n} \rightarrow 0
$$

so that now

$$
a_{n \nu}=\left(1-\cos \nu \theta_{n}\right) / \nu^{2} \theta_{n}, \quad \nu=1,2, \cdots, n .
$$

Evidently $0<a_{n \nu} \rightarrow 0$ as $n \rightarrow \infty$; hence a necessary and sufficient condition that for every convergent sequence $\tau_{n} \rightarrow \tau$ the sequence $S_{n}$ has a limit $\sigma \tau$ is that $\lim \sum_{1}^{n} A_{n \nu}=\sigma$ exists (from Theorem A). Now

$$
\begin{aligned}
\sum_{1}^{n} \frac{1-\cos \nu \theta_{n}}{\nu^{2}} & =\int_{0}^{\theta_{n}}\left(\sum_{1}^{n} \frac{\sin \nu t}{\nu}\right) d t \\
& =\int_{0}^{\theta_{n}}\left\{-\frac{t}{2}+\int_{0}^{t} \frac{\sin (n+1 / 2)^{u}}{2 \sin (u / 2)} d \kappa\right\} d t \\
& =-\frac{\theta_{n}^{2}}{4}+\int_{0}^{\theta_{n}}\left(\theta_{n}-t\right) \frac{\sin (n+1 / 2) t}{2 \sin (t / 2)} d t .
\end{aligned}
$$


thus

$$
\begin{aligned}
\sum_{1}^{n} a_{n v}= & -\frac{\theta_{n}^{2}}{4}+\theta_{n}^{-1} \int_{0}^{\theta_{n}}\left(\theta_{n}-t\right) \frac{\sin (n+1 / 2) t}{t} d t \\
& +\theta_{n}^{-1} \int_{0}^{\theta_{n}}\left(\theta_{n}-t\right)\left\{\frac{1}{2 \sin (t / 2)}-\frac{1}{t}\right\} \sin (n+1 / 2) t d t
\end{aligned}
$$

and using (3.5)

$$
\begin{aligned}
\theta_{n}^{-1}\left|\int_{0}^{\theta_{n}}\left(\theta_{n}-t\right)\left\{\frac{1}{2 \sin (t / 2)}-\frac{1}{t}\right\} \sin (2 n+1) \frac{t}{2} d t\right| \\
<\theta_{n}^{-1} \int_{0}^{\theta_{n}}\left(\theta_{n}-t\right) \frac{t d t}{12}<\frac{1}{24} \theta_{n}^{2} \rightarrow 0 .
\end{aligned}
$$

Hence

$$
\begin{aligned}
\sum_{1}^{n} a_{n \nu} & =o(1)+\theta_{n}^{-1} \int_{0}^{(n+1 / 2) \theta_{n}}\left(\theta_{n}-\frac{t}{n+1 / 2}\right) \frac{\sin t}{t} d t \\
& =o(1)+\int_{0}^{(n+1 / 2) \theta_{n}} \frac{\sin t}{t} d t-\frac{2}{(2 n+1) \theta_{n}}\left[1-\cos (n+1 / 2) \theta_{n}\right] .
\end{aligned}
$$

If $\beta \leqq \infty$ is a limit point of the sequence $\left\{n \theta_{n}\right\}$, then for a subsequence of integers $n$

$$
\sum_{1}^{n} a_{n \nu} \rightarrow \int_{0}^{\beta} \frac{\sin t}{t} d t-\frac{1-\cos \beta}{\beta} \equiv g(\beta) .
$$

Now $g^{\prime}(\beta)=(1-\cos \beta) / \beta^{2} \geqq 0$, hence $g(\beta) \uparrow$, as $\beta$ increases, to $g(\infty)=\pi / 2$. We have thus proved:

THEOREM 5. In order that for every convergent sequence $\tau_{n} \rightarrow \tau$ the transform $S_{n}=\sum_{1}^{n} \tau_{\nu}\left(1-\cos \nu \theta_{n}\right) / \nu^{2} \theta_{n}$ has a limit, it is necessary and sufficient that $\lim _{n \rightarrow \infty} \theta_{n}=\beta, \beta \leqq \infty$, exists. We then have $S_{n} \rightarrow \tau g(\beta)$, where $g(\beta)=\int_{0}^{\beta}\left(t^{-1} \sin t\right) d t$ $-(1-\cos \beta) / \beta \leqq \pi / 2$.

We now assume only $n^{-1} \sum_{1}^{n} \tau_{\nu} \rightarrow \tau$, or $n^{-1} \tau_{n}^{\prime} \rightarrow \tau$. We have $S_{n}=\sum_{1}^{n-1}\left(a_{n}\right.$, $\left.-a_{n, \nu+1}\right) \tau_{\nu}^{\prime}+a_{n n} \tau_{n}^{\prime}$; and the additional condition for the existence of lim $S_{n}$ is by Theorem $\mathrm{A}$ :

$$
\sum_{1}^{n-1} \nu\left|\frac{1-\cos \nu \theta_{n}}{\nu^{2} \theta_{n}}-\frac{1-\cos (\nu+1) \theta_{n}}{(\nu+1)^{2} \theta_{n}}\right|+\frac{1-\cos n \theta_{n}}{n \theta_{n}}=O(1),
$$

or

$$
\theta_{n}^{-1} \sum_{1}^{n-1} \nu^{-3}\left|(\nu+1)^{2}\left(1-\cos \nu \theta_{n}\right)-\nu^{2}\left(1-\cos (\nu+1) \theta_{n}\right)\right|=O(1) .
$$


But

$$
\theta_{n}^{-1} \sum_{1}^{n} \nu^{-3}(2 \nu+1)\left(1-\cos \nu \theta_{n}\right)<3 \sum_{1}^{n} \frac{1-\cos \nu \theta_{n}}{\nu^{2} \theta_{n}}=3 \sum_{1}^{n} a_{n \nu}=O(1),
$$

hence (4.3) reduces to $\theta_{n}^{-1} \sum_{1}^{n-1} \nu^{-1}\left|\cos \nu \theta_{n}-\cos (\nu+1) \theta_{n}\right|=\dot{O}(1)$, as $n \rightarrow \infty$, or $\sum_{1}^{n} \nu^{-1}\left|\sin (\nu+1 / 2) \theta_{n}\right|=O(1)$.

But this condition is equivalent to $n \theta_{n}=O(1)$ (cf. $[3, \S 2]$ ). Thus we have the following:

TheOREM 6. The transform $\left.S_{n}=\sum_{1}^{n} \tau_{\nu}\left(1-\cos \nu \theta_{n}\right) / \nu^{2} \theta_{n}\right)$ has a limit for every sequence $\left\{\tau_{n}\right\}$ which is summable $(C, 1)$ to $\tau$, if and only if $\lim n \theta_{n}=\beta<\infty$ exists. We then have $S_{n} \rightarrow \tau g(\beta)$.

We finally assume $(C, 2) \lim \tau_{n}=\tau$; using the formula

$$
\sum_{1}^{n} a_{\nu} \tau_{\nu}=\sum_{1}^{n-2} \tau_{\nu}^{2} \Delta^{2} a_{\nu}+\tau_{n-1}^{2}\left(a_{n-1}-2 a_{n}\right)+\tau_{n}^{2} a_{n},
$$

we now get for the existence of $\lim S_{n}$ the additional conditions

(4.4) $\theta_{n}^{-1} \sum_{1}^{n-2} \nu^{2}\left|\frac{1-\cos \nu \theta_{n}}{\nu^{2}}-2 \frac{1-\cos (\nu+1) \theta_{n}}{(\nu+1)^{2}}+\frac{1-\cos (\nu+2) \theta_{n}}{(\nu+2)^{2}}\right|=O(1)$,

$$
\theta_{n}^{-1} n^{2}\left|\frac{1-\cos (n-1) \theta_{n}}{(n-1)^{2}}-2 \frac{1-\cos n \theta_{n}}{n^{2}}\right|+\frac{1-\cos n \theta_{n}}{\theta_{n}}=O(1) \text {. }
$$

In particular $\sin ^{2} n \theta_{n} / 2=O\left(\theta_{n}\right)=o(1)$, hence $n \theta_{n} \rightarrow \beta=2 \lambda \pi, \lambda$ an integer. We assume $\lambda>0$; on putting $n \theta_{n}=2 \lambda \pi+2 \epsilon_{n}, \epsilon_{n} \rightarrow 0$, we must have

$$
\sin ^{2}\left(\lambda \pi+\epsilon_{n}\right)=\sin ^{2} \epsilon_{n}=O\left(\left(\lambda \pi+\epsilon_{n}\right) / n\right)=O(1 / n),
$$

or $\epsilon_{n}=O\left(1 / n^{1 / 2}\right)$. To satisfy (4.5) the additional condition is $\sin ^{2}(n-1) \theta_{n}$ $=O\left(\theta_{n}\right)$, or $\left(\sin n \theta_{n} \cos \theta_{n}-\cos n \theta_{n} \sin \theta_{n}\right)^{2}=O(1 / n)$, which reduces to our previous condition. We finally show that now (4.4) is also satisfied. We have

hence

$$
\frac{1-\cos \nu \theta}{\nu^{2}}=\int_{0}^{\theta} d t \int_{0}^{t} \cos \nu u d u=\int_{0}^{\theta}(\theta-t) \cos \nu t d t
$$

$$
\Delta^{2} \frac{1-\cos \nu \theta}{\nu^{2}}=\int_{0}^{\theta}(\theta-t) \Delta^{2} \cos \nu t d t=R \int_{0}^{\theta}(\theta-t) z^{\nu}(1-z)^{2} d t, \quad z=e^{i t},
$$

and

$$
\left|\Delta^{2} \frac{1-\cos \nu \theta}{\nu^{2}}\right|<\int_{0}^{\theta}(\theta-t)\left|1-e^{i t}\right|^{2} d t<\int_{0}^{\theta}(\theta-t) t^{2} d t=\frac{\theta^{4}}{12} .
$$


Using this inequality, the left side of (4.4) is less than $\sum_{1}^{n-2} \nu^{2} \theta_{n}^{3}<n^{3} \theta_{n}^{3}=O(1)$. This proves the following theorem.

THEOREM 7. The transform $S_{n}$ has a limit $\sigma \tau$ (with $\sigma \neq 0$ ) for every sequence $\left\{\tau_{n}\right\}$ which is summable $(C, 2)$ to $\tau$ if and only if $n \theta_{n}=2\left(\lambda \pi+\epsilon_{n}\right), \epsilon_{n}=O\left(n^{-1 / 2}\right)$, $\lambda$ an integer greater than 0 . We then have $S_{n} \rightarrow \tau \int_{0}^{2 \lambda \pi} t^{-1} \sin t d t=\tau \sigma(2 \lambda \pi)$.

5. $(C, 2)$ summability of $\left\{n b_{n}\right\}$ for sine series. We shall prove the following theorem.

THEOREM 8. If $f(\theta) \sim \sum_{1}^{\infty} b_{\nu} \sin \nu \theta$, and for some $j$

$$
\int_{0}^{\theta}|f(t)-j / 2| d t=O(\theta), \int_{0}^{\theta}\{f(t)-j / 2\} d t=o(\theta) \text {, as } \theta \downarrow 0,
$$

then

$$
(C, 2) \lim n b_{n}=j / \pi
$$

We have, for $\tau_{n}=n b_{n}$,

$$
\tau_{n}^{2}=\sum_{1}^{n}(n-\nu+1) \nu b_{\nu}=\frac{2}{\pi} \int_{0}^{\pi} f(t)\left(\sum_{1}^{n}(n-\nu+1) \nu \sin \nu t\right) d t,
$$

and $\sum_{1}^{n}(n-\nu+1) \nu \sin \nu t=-(d / d t) \sum_{1}^{n}(n-\nu+1) \cos \nu t$. On putting

$$
\sum_{1}^{n} \cos \nu t=-\frac{1}{2}+\frac{\sin (n+1 / 2) t}{2 \sin (t / 2)} \equiv \sigma_{n}(t),
$$

we have

$$
\begin{aligned}
\sum_{1}^{n}(n-\nu+1) \cos \nu t & =\sum_{1}^{n} \sigma_{\nu}(t)=-\frac{n}{2}+\frac{1}{2 \sin (t / 2)} \sum_{1}^{n} \sin (\nu+1 / 2) t \\
& =-\frac{n}{2}+\frac{1}{2 \sin (t / 2)}\left\{\sin ^{2}(n+1)(t / 2)-\sin ^{2}(t / 2)\right\} \\
& =-\frac{n+1}{2}+\frac{1}{2} \cdot \frac{\sin ^{2}(n+1)(t / 2)}{\sin ^{2}(t / 2)}
\end{aligned}
$$

hence

$$
\begin{aligned}
& \sum_{1}^{n}(n-\nu+1) \nu \sin \nu t \\
& =\frac{1}{2} \frac{\sin (n+1)(t / 2)}{\sin ^{3}(t / 2)}\left\{\cos \frac{t}{2} \sin (n+1) \frac{t}{2}-(n+1) \cos (n+1) \frac{t}{2} \sin \frac{t}{2}\right\} .
\end{aligned}
$$

Thus 


$$
\tau_{n}^{2}=\frac{1}{\pi} \int_{0}^{\pi} f(t) \frac{\sin (n+1)(t / 2)}{\sin ^{3}(t / 2)}\left\{\sin n \frac{t}{2}-n \cos (n+1) \frac{t}{2} \sin \frac{t}{2}\right\} d t
$$

furthermore

$$
\begin{aligned}
& \int_{0}^{\pi}\left(\sum_{1}^{n}(n-\nu+1) \nu \sin \nu t\right) d t \\
& =\sum_{1}^{n}(n-\nu+1)\{1-(-1) \nu\}=2 \sum_{\lambda=0}^{(n-1) / 2}(n-2 \lambda) \\
& =4 \sum_{\lambda=0}^{(n-1) / 2}\left(\frac{n}{2}-\lambda\right)=h_{n},
\end{aligned}
$$

say, where $2 h n / n^{2} \rightarrow 1$ as $n \rightarrow \infty$. Our aim is to prove

$$
\tau_{n}^{2} / h_{n} \rightarrow j / \pi, \quad \text { or } \quad\left(\tau_{n}^{2}-j h_{n} / \pi\right) / h_{n} \rightarrow 0 .
$$

From (5.3), (5.4) and (5.5)

$$
\tau_{n}^{2}-\frac{1}{\pi} j h_{n}=\frac{1}{\pi} \int_{0}^{\pi}\{f(t)-j / 2\} \frac{\sin (n+1)(t / 2)}{\sin ^{3}(t / 2)} K_{n}(t) d t,
$$

where

$$
\begin{aligned}
K_{n}(t) & =\sin (n t / 2)-n \cos ((n+1) t / 2) \sin (t / 2) \\
& =n \sin (t / 2)(1-\cos ((n+1) t / 2))-(n \sin (t / 2)-\sin (n t / 2))
\end{aligned}
$$

Now from (3.5)

$$
\begin{aligned}
0<\frac{1}{\sin ^{3}(t / 2)}-\frac{1}{(t / 2)^{3}} & =\left(\frac{1}{\sin (t / 2)}-\frac{2}{t}\right)\left(\frac{1}{\sin ^{2}(t / 2)}+\frac{2}{t \sin (t / 2)}+\frac{4}{t^{2}}\right) \\
& <\frac{t}{6} \cdot \frac{3}{\sin ^{2}(t / 2)}<\frac{\pi}{2 \sin (t / 2)},
\end{aligned}
$$

and

$$
\left|K_{n}(t)\right|<n \sin (t / 2)+|\sin n(t / 2)| \text {. }
$$

Hence

$$
\begin{aligned}
\mid \int_{0}^{\pi}\{f(t)-j / 2\} & \left(\frac{1}{\sin ^{3}(t / 2)}-\frac{1}{(t / 2)^{3}}\right) \sin (n+1) \frac{t}{2} K_{n}(t) d t \mid \\
& <\frac{\pi}{2} \int_{0}^{\pi}|f(t)-j / 2|\left(n+\frac{|\sin (n t / 2)|}{\sin (t / 2)}\right) d t=O(n)=o\left(h_{n}\right) ;
\end{aligned}
$$

thus (5.6) will follow if we prove 


$$
\int_{0}^{\pi}\{f(t)-j / 2\} \frac{\sin ((n+1) t / 2)}{t^{3}} K_{n}(t) d t=o\left(n^{2}\right) .
$$

In view of (5.7) this will follow from

$$
\int_{0}^{\pi}\{f(t)-j / 2\} \frac{\sin ((n+1) t / 2)}{t^{3}} \sin \frac{t}{2}\left(1-\cos (n+1) \frac{t}{2}\right) d t=o(n)
$$

and

$$
\int_{0}^{\pi}\{f(t)-j / 2\} \frac{\sin ((n+1) t / 2)}{t^{3}}\left(n \sin \frac{t}{2}-\sin n \frac{t}{2}\right) d t=o\left(n^{2}\right)
$$

Again, using (3.5),

$$
\begin{array}{r}
\left|\int_{0}^{\pi}\{f(t)-j / 2\} \frac{\sin ((n+1) t / 2)}{t^{3}}\left(\frac{t}{2}-\sin \frac{t}{2}\right)\left(1-\cos (n+1) \frac{t}{2}\right) d t\right| \\
<\int_{0}^{\pi}|f(t)-j / 2| d t=O(1),
\end{array}
$$

thus (5.8) is equivalent to

$$
\int_{0}^{\pi}\{f(t)-j / 2\} \frac{\sin ((n+1) t / 2)}{t^{2}}\left(1-\cos (n+1) \frac{t}{2}\right) d t=o(n),
$$

or

$$
I_{n} \equiv \int_{0}^{\pi}\{f(t)-j / 2\} \frac{\sin ((n+1) t / 2)-2^{-1} \sin (n+1) t}{t^{2}} d t=o(n) .
$$

We write $I_{n}=\int_{0}^{c / n}+\int_{c / n}^{\pi}=L_{1}+L_{2}$, say, where $c$ is a constant, arbitrarily large. On putting $\int_{0}^{\theta}\{f(t)-j / 2\} d t=F(\theta)$, and using integration by parts, we get from (5.1)

$$
\begin{aligned}
L_{1}= & \left.\frac{F(t)}{t} \frac{\sin (n+1)(t / 2)-2^{-1} \sin (n+1) t}{t}\right]_{0}^{c / n} \\
& -(n+1) \int_{0}^{c / n} F(t) \frac{\cos ((n+1) t / 2)-\cos (n+1) t}{2 t^{2}} d t \\
& +2 \int_{0}^{c / n} F(t) \frac{\sin ((n+1) t / 2)-2^{-1} \sin (n+1) t}{t^{3}} d t \\
= & o(n)+o(n) \cdot \int_{0}^{c / n} t^{-1}|\sin (3(n+1) t / 4) \sin ((n+1) t / 4)| d t \\
& +o(1) \int_{0}^{c / n} t^{-2} \sin ^{2}((n+1) t / 4) d t=o(n),
\end{aligned}
$$


as $n \rightarrow \infty$. Furthermore $\left|L_{2}\right|<\int_{c / n}^{\pi}|f(t)-j / 2| 2 \sin ^{2}((n+1) t / 4) / t^{2} d t$; we use here Fejér's inequality, $\sin ^{2} x<(2 x /(1+x))^{2}$ for $x>0$. Then

$$
\begin{aligned}
\left|L_{2}\right|< & \frac{(n+1)^{2}}{2} \int_{c / n}^{\pi}|f(t)-j / 2| \frac{d t}{(1+(.(n+1) t / 4))^{2}} \\
= & \left.\frac{(n+1)^{2}}{2} \frac{\phi(t)}{(1+((n+1) t / 4))^{2}}\right]_{c / n}^{\pi} \\
& +\frac{(n+1)^{2}}{2} \int_{c / n}^{\pi} \frac{(n+1) \phi(t) d t}{4(1+((n+1) t / 4))^{3}}
\end{aligned}
$$

where $\phi(\theta)=\int_{0}^{\theta}|f(t)-j / 2| d t$. But from (5.1), $\phi(\theta)<\gamma \theta, \gamma$ an absolute constant, hence

$$
\begin{aligned}
\left|L_{2}\right| & <O(1)+\frac{(n+1)^{3}}{8} \gamma \int_{c / n}^{\pi} \frac{t d t}{(1+((n+1) t / 4))^{3}} \\
& <O(1)+\frac{(n+1)^{2}}{2} \gamma \int_{c / n}^{\pi} \frac{d t}{(1+((n+1) t / 4))^{2}}<\frac{2 \gamma(n+1)}{1+c / 4}+O(1),
\end{aligned}
$$

and $\lim \sup _{n \rightarrow \infty} n^{-1} L_{2} \leqq 8 \gamma /(4+c)$. Thus lim $\sup _{n \rightarrow \infty} n^{-1}\left|I_{n}\right| \leqq 8 \gamma /(4+c)$; but $c$ being arbitrary, now (5.8) follows.

Similarly we decompose the left side of (5.9) into $\int_{0}^{c / n}+\int_{c / n}^{\pi}=M_{1}+M_{2}$ say, where

$$
\begin{aligned}
M_{1}= & \int_{0}^{c / n}\{f(t)-j / 2\} t^{-3}\left[\frac{n}{2}\left(\cos n \frac{t}{2}-\cos (n+2) \frac{t}{2}\right)\right. \\
& \left.+\frac{1}{2}\left(\cos (2 n+1) \frac{t}{2}-\cos \frac{t}{2}\right)\right] d t \\
\equiv & \int_{0}^{c / n}\{f(t)-j / 2\} t^{-3} Q_{n}(t) d t \\
= & \left.F(t) t^{-3} Q_{n}(t)\right]_{0}^{c / n}-\int_{0}^{c / n} F(t) t^{-3} Q_{n}^{\prime}(t) d t \\
& +3 \int_{0}^{c / n} t^{-4} Q_{n}(t) F(t) d t .
\end{aligned}
$$

The mean value theorem yields

$$
\frac{Q_{n}(t)}{n^{4} t^{4}} \sim \frac{Q_{n}^{\prime}(t)}{4 n^{4} t^{3}} \sim \frac{Q_{n}^{\prime \prime}(t)}{12 n^{4} t^{2}} \sim \frac{Q^{\prime \prime \prime}(t)}{24 n^{4} t} \sim \frac{1}{96}
$$

for $t \rightarrow 0$, hence, using (5.1), we find that $M_{1}=o\left(n^{2}\right)$, as $n \rightarrow \infty$. 
To estimate $M_{2}$ we use the formula

$$
n \sin x-\sin n x=\sin x \sum_{\nu=1}^{n}\{1-\cos (n-2 \nu+1) x\}>0, \quad 0<x<\pi,
$$

which follows from

$$
\frac{\sin n x}{\sin x}=\frac{e^{i n x}-e^{-i n x}}{e^{i x}-e^{-i x}}=\sum_{1}^{n} e^{i(n-2 \nu+1) x}=\sum_{1}^{n} \cos (n-2 \nu+1) x .
$$

Now

$$
\begin{aligned}
\left|M_{2}\right| & <\int_{c / n}^{\pi}|f(t)-j / 2| t^{-3} \sin \frac{t}{2} \sum_{1}^{n}\left\{1-\cos (n-2 \nu+1) \frac{t}{2}\right\} d t \\
& <\int_{c / n}^{\pi}|f(t)-j / 2| t^{-2} \sum_{2 \nu \leqq n}\left\{1-\cos (n-2 \nu+1) \frac{t}{2}\right\} d t=\sum_{2 \nu \leqq n} D_{\nu}
\end{aligned}
$$

say, and, as in the estimate of $L_{2}$

$$
\begin{aligned}
D_{\nu} & <\phi(\pi) \frac{(n-2 \nu+1)^{2}}{2(1+(n-2 \nu+1) \pi / 4)^{2}}+\frac{2 \gamma(n-2 \nu+1)}{1+c / 4} \\
& <\phi(\pi)+\frac{8 \gamma(n-2 \nu+1)}{4+c} .
\end{aligned}
$$

Hence $\left|M_{2}\right|<n \phi(\pi)+4 \gamma n^{2} /(4+c)$, and lim $\sup _{n \rightarrow \infty}\left|M_{2}\right| / n^{2} \leqq 4 \gamma /(4+c) ; c$ being arbitrarily large, we finally get (5.9), and Theorem 8 is proved.

6. The jump of $f(\theta)$ and Gibbs' phenomenon. The foregoing results enable us to give new formulae for the jump of $f(\theta)$ and to prove a Gibbs phenomenon.

THEOREM 9. Under the assumptions (5.1), $j$ is determined by any one of the formulae

$$
\begin{aligned}
(C, 2) \lim n b_{n} & =\frac{1}{\pi} j, \\
\sum_{1}^{n} b_{\nu} \sin \nu \frac{\pi}{n} & \rightarrow \frac{1}{\pi} j \int_{0}^{\pi} \frac{\sin t}{t} d t, \\
\lim _{n \rightarrow \infty} \frac{1}{n} \sum_{1}^{n} s_{\nu}\left(\frac{\pi}{\nu}\right) & =\frac{1}{\pi} j \int_{0}^{\pi} \frac{\sin t}{t} d t, \\
\lim \frac{n}{2 \pi} \int_{0}^{2 \pi / n} s_{n}(t) d t & =\frac{1}{\pi} j \int_{0}^{2 \pi} \frac{\sin t}{t} d t,
\end{aligned}
$$

where $s_{n}(t)=\sum_{1}^{n} b_{,} \sin \nu t$. 
(6.1) is the statement of Theorem 8; (6.2) follows from Theorem 2 for $\theta_{n} \equiv 0, \phi_{n}=\pi / n, \tau_{n}=n b_{n}$, using (6.1). Similarly (6.3) follows from Theorem 4 for $\tau_{n}=n b_{n}, \theta_{n}=\pi / n$, and (6.4) follows from Theorem 7 for $\theta_{n}=2 \pi / n$.

To prove a Gibbs' phenomenon under the same assumptions, put, in Theorem $2, \tau_{n}=n b_{n}, \theta_{n} \equiv 0, n \phi_{n}=\pi+O(1 / n)$, then

hence

$$
s_{n}\left(\phi_{n}\right) \rightarrow \frac{1}{\pi} j \int_{0}^{\pi} t^{-1} \sin t d t
$$

$$
\lim \sup s_{n}\left(\theta_{n}\right) \geqq j / 2 \times 1.08949 \cdots, \quad \theta_{n} \downarrow 0 .
$$

Similarly from Theorem 4 for $\alpha=\pi$

$$
\frac{1}{n} \sum_{1}^{n} s_{\nu}\left(\theta_{\nu}\right) \rightarrow \frac{1}{\pi} j \int_{0}^{\pi} t^{-1} \sin t d t
$$

hence

$$
\lim \sup s_{n}\left(\theta_{n}\right) \geqq \frac{1}{\pi} j \int_{0}^{\pi} t^{-1} \sin t d t
$$

Theorem 7 also proves the presence of a Gibbs' phenomenon, however with a smaller constant.

We may also combine Theorem 8 with Theorem 3 of [3], putting there $\rho_{n} \equiv 1, \kappa=2, n \theta_{n}=\pi+O\left(n^{-1}\right)$. Summarizing, we have the following theorem.

THEOREM 10. Under the assumptions (5.1)

where

$$
\limsup _{\theta_{n} \downarrow 0} \sum_{1}^{n} b_{\nu} \sin \nu \theta_{n} \geqq \frac{j g}{2},
$$

$$
g=\frac{2}{\pi} \int_{0}^{\pi} t^{-1} \sin t d t=1.08949 \ldots
$$

\section{LITERATURE}

1. W. Rogosinski, Über die Abschnitte trigonometrischer Reihen, Math. Ann. vol. 95 (1926) pp. 110-134.

2. W. Rogosinski, Über den Einfluss einseitiger Eigenschaften einer Funktion auf ihre Fourierreihe, Schriften der Königsberger Gelehrten Gesellschaft vol. 3 (1926) pp. 57-98.

3. O. Szász, On the partial sums of Fourier series at points of discontinuity, Trans. Amer. Math. Soc. vol. 53 (1943) pp. 440-453.

University of Cincinnati, Cincinnati, Ohio. 